\title{
Stokes flow of micropolar fluid past a viscous fluid spheroid with non-zero boundary condition for microrotation
}

\author{
M KRISHNA PRASAD*D and MANPREET KAUR \\ Department of Mathematics, National Institute of Technology, Raipur 492010, India \\ e-mail: madaspra.maths@nitrr.ac.in; kpm973@gmail.com; manpreet.kaur22276@yahoo.com
}

MS received 21 June 2015; revised 29 April 2016; accepted 23 May 2016

\begin{abstract}
The Stokes axisymmetric flow of an incompressible micropolar fluid past a viscous fluid spheroid whose shape deviates slightly from that of a sphere is studied analytically. The boundary conditions used are the vanishing of the normal velocities, the continuity of the tangential velocities, continuity of shear stresses and spin-vorticity relation at the surface of the spheroid. The hydrodynamic drag force acting on the spheroid is calculated. An exact solution of the problem is obtained to the first order in the small parameter characterizing the deformation. It is observed that due to increased spin parameter value, the drag coefficient decreases. Wellknown results are deduced and comparisons are made with classical viscous fluid and micropolar fluids.
\end{abstract}

Keywords. Stokes flow; micropolar fluid; drag force; spheroid; spin-vorticity relation.

\section{Introduction}

The motion of a fluid droplet in a second immiscible fluid through a continuous medium at low Reynolds numbers is of much interest in the fields of chemical, biomedical and environmental engineering and science. This study plays an important role in natural and industrial processes such as raindrop formation, the mechanics and rheology of emulsions, liquid-liquid extraction, motion of blood cells in an artery or vein, extraction of crude oil from petroleum products and sedimentation phenomena. The problem of resistance on a moving body due to a medium is a classical and well-studied problem. The creeping flow motion of a single spherical drop of radius $a$ in an unbounded medium of viscosity $\mu$ was first analysed independently by Hadamard [1] and Rybczynski [2]. Assuming continuous velocity and continuous tangential shear stress across the interface between the fluid phases in the absence of surface active agents, they found that the force exerted on the fluid sphere by the surrounding fluid is

$$
F=-6 \pi \mu a U \frac{3 \mu^{*}+2}{3 \mu^{*}+3}
$$

where $U$ is the migration velocity of the drop and $\mu^{*}$ is the internal to external viscosity ratio. The above formula reduces to the drag force exerted on a no-slip solid sphere (Stokes' law) when the viscosity of the drop is infinite and to the case of a perfect-slip gas bubble when the viscosity approaches zero.

\footnotetext{
*For correspondence
}

In many practical applications of low Reynolds number motion of fluid droplets, the hydrodynamics of classical fluids is based on the assumption that the fluid particles do not have any internal structure. However, there are cases of industrial significance where one of the immiscible fluids is non-Newtonian and fluid particles may exhibit microscopical effects such as rotation and shrinking. A well-accepted theory that accounts for internal structures of fluids is the micropolar fluid theory by Eringen [3, 4]. These fluids consist of rigid, randomly oriented particles with their own spins and microrotations suspended in a viscous medium. There are two kinematics vector fields: the usual velocity field and an axial vector that represents the spin or the microrotation of the micropolar fluid particles, which are assumed to be rigid. The review article by Ariman et al $[5,6]$ describes some of the various applications of miropolar fluids that have been explored. The mathematical theory of equations of micropolar fluids and applications of these fluids in the theory of lubrication and in the theory of porous media are dealt with in a recent book by Lukaszewicz [7].

Hetsroni and Haber [8] discussed the problem of a single spherical droplet submerged in an unbounded viscous fluid of a different viscosity. They assumed the droplet to be spherical as a first approximation and later determined the equation of the interface using an iterative method. Using spherical bipolar coordinates, Bart [9] examined the motion of a spherical droplet settling normal to a plane interface between two immiscible viscous fluids. He obtained the exact solution by assuming that the sphere moves perpendicularly to the plane at low Reynolds number conditions. Wacholder and Weihs [10] also utilized bipolar coordinates 
to study the motion of a fluid sphere through another fluid normal to a rigid or free plane surface and their calculations agree with the results obtained by Bart. They obtained exact solutions for the forces on a fluid particle in the presence of another fluid particle when both are falling along their lineof-centres, or of a plane surface normal to the settling velocity vector. Lee and Keh [11] employed a combined analytical-numerical method with the boundary collocation technique to examine the quasisteady creeping flow of a spherical drop in an immiscible fluid within a spherical cavity. They obtained the wall-corrected drag force exerted on the drop with good convergence. Recently, Choudhuri and Sri Padmavati [12] studied an arbitrary unsteady Stokes flow in and around a liquid sphere. They assumed the shape of the liquid drop to be spherical as a first approximation and used an iterative procedure to determine the shape of the deformed liquid sphere as done by Hetsroni and Haber [8]. They have also discussed the flow inside and outside the liquid sphere generated due to an oscillating singularity inside the sphere and found that the deformation is mainly affected by two parameters: the ratio of the viscosities of the two fluids and the capillary number. Tripathi et al [13] noticed that the falling drop does not in general behave like a rising bubble. They have shown that the mechanism that differentiates the dynamics of a bubble and a drop is as follows: the vorticity tends to concentrate in the lighter fluid, and this affects the entire dynamics. They numerically found a similarity in bubble and drop dynamics for moderate values of surface tension and inertia, so long as the density ratio is close to unity. Tripathi et al [14] studied the three-dimensional simulation of a rising bubble in a denser and more viscous fluid under the action of buoyancy. They used free-slip and no-penetration conditions on all the boundaries and found that as the size of the bubble increases, the dynamics goes through three sudden transitions from one known class of shapes to another.

All results cited above concern viscous fluids. For micropolar fluids, the Stokes flow of micropolar fluid past a rigid sphere, spheroid and approximate sphere was considered by Rao and Rao [15], Rao and Iyengar [16] and Iyengar and Srinivasacharya [17], respectively. Rao and Rao [15] found that the drag on the sphere is more than in the case of non-polar fluids. Rao and Iyengar [16] found the expressions for the velocity, microrotation, stress and couple stress analytically in an infinite series form. They evaluated the drag on the body and observed that there is no couple exerted on the body. Iyengar and Srinivasacharya [17] evaluated the drag on an oblate spheroid and found that the drag force on the spheroid is less than on a sphere of radius equal to the equatorial radius of the spheroid. Drag on an axially symmetric body in Stokes flow of a micropolar fluid has been evaluated by Ramkissoon and Majumdar [18] and they observed that the drag in the micropolar fluid is greater than that in the classical fluid. Ramkissoon [19] has obtained the solution for the problem of a micropolar fluid flow around a Newtonian fluid sphere and evaluated drag force exerted on the sphere. He determined the flow field within the Newtonian fluid sphere and of the micropolar fluid outside the sphere and evaluated drag force exerted on the sphere. Ramkissoon and Majumdar [20] extended the work of Ramkissoon [19] to a spheroid whose shape varies slightly from that of a sphere. They consider the micropolar flow past an oblate fluid spheroid and determine the drag experienced by it. Hayakawa [21] has studied the problem of axisymmetric slow viscous flow of a micropolar fluid past a stationary sphere and a stationary cylinder explicitly, and computed the drag force in each case. Hoffmann et al [22] calculated the resistance force exerted on a sphere moving with a constant velocity in a micropolar fluid using non-zero boundary condition for microrotation. The problems of the flow of a viscous fluid past a micropolar fluid sphere and the flow of a micropolar fluid past a viscous fluid drop have been discussed by Niefer and Kaloni [23] with non-zero spin boundary condition. They obtained the expression for drag in each case and found that the viscosity ratios and the spin parameter have significant effect upon the drag in each case. Saad [24] discussed the problem of flow of micropolar fluid past a viscous fluid sphere and the flow of a viscous fluid past a micropolar fluid sphere using non-zero spin boundary condition on fluid-fluid interface and four known boundary conditions: Happel, Kuwabara, Kvashnin and Cunningham (Mehta Morse condition) on the cell surface. $\mathrm{He}$ evaluated the normalized hydrodynamic drag force exerted on the spherical droplet in each case. The problem of the creeping flow of an incompressible micropolar fluid past a fluid sphere with non-zero boundary condition for microrotation is analysed by Satya and Pankaj [25]. They evaluated the drag force experienced by the fluid sphere. The problem of creeping flow of an incompressible micropolar fluid past a Reiner-Rivlin liquid sphere with non-zero boundary condition for microrotations was investigated by Jaiswal and Gupta [26]. They used a nonhomogeneous boundary condition for the microrotation vector and evaluated the drag force exerted on ReinerRivlin liquid sphere. Faltas and Saad [27] employed a combined analytical-numerical method with the boundary collocation technique to examine the Stokes flow of a viscous fluid sphere immersed in a micropolar fluid and moving perpendicular to a planar solid surface as well as for the related problem of a micropolar fluid sphere immersed in a viscous fluid. They solved the problem using the no-slip and no-spin boundary conditions at the impermeable plane surface and evaluated the drag in each case with good convergence.

The aim of this paper is to calculate the resistance force exerted on a viscous fluid spheroid whose shape deviates slightly from that of a sphere, moving with a uniform velocity in a micropolar fluid by applying non-zero boundary condition for microrotation vector and to estimate the influence of the microrotation on the motion of fluid spheroidal particle in micropolar fluid. To determine the 
way in which the drag acting on the fluid spheroid is influenced by the fluid flow parameters, the variation of drag force with viscosity ratio, spin parameter, micropolarity parameter and deformation parameter is presented graphically and discussed. Some previous well-known results are also deduced from the present analysis.

\section{Formulation of the problem}

Consider a fluid spheroid moving into an unbounded volume of another immiscible micropolar fluid. The following assumptions are considered to be valid:

1. the fluid inside the particle is Newtonian and the fluid in the surrounding medium is micropolar,

2. the flows are steady and axisymmetric,

3 . the physical properties (density $\rho$ and viscosities $\left.\alpha_{0}, \beta_{0}, \gamma_{0}, \kappa, \mu^{(1)}, \mu^{(2)}\right)$ of the particle and medium are constant,

4. the shape of the particle deforms slightly from that of a sphere,

5. there is no interfacial mass transfer (the radial velocity is zero at the interface) and

6. there are no surface-active materials.

Consider the steady axisymmetric flow of an incompressible micropolar fluid past a viscous fluid spheroid that is held fixed in a uniform stream of velocity $U$ (figure 1). The external region and the internal region are denoted by regions I and II, respectively. The equations of motion for the region outside the spheroid are the equations governing the steady flow of an incompressible micropolar fluid under Stokesian assumption with the absence of body force and body couple and are given by [4]

$$
\nabla \cdot \mathbf{q}^{(1)}=0
$$

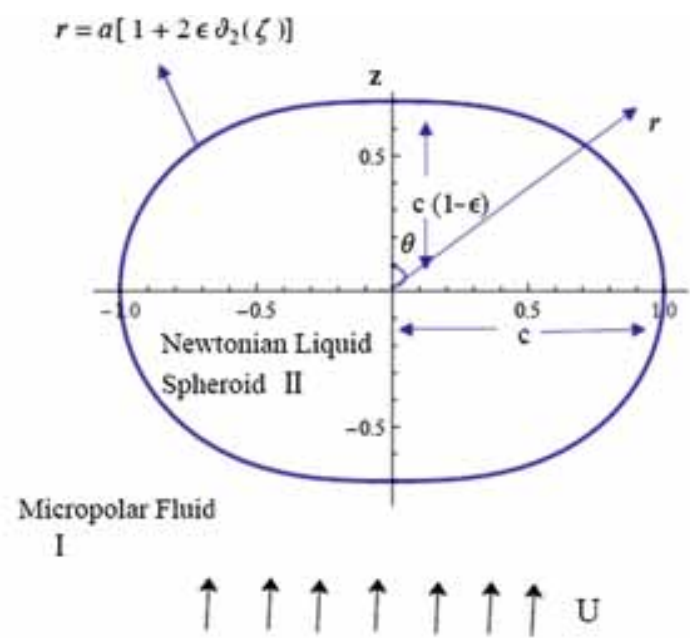

Figure 1. The physical situation and the coordinate system.

$$
\begin{gathered}
\nabla p^{(1)}+\left(\mu_{1}+\kappa\right) \nabla \times \nabla \times \mathbf{q}^{(1)}-\kappa \nabla \times \mathbf{v}=0 \\
\kappa \nabla \times \mathbf{q}^{(1)}-2 \kappa \boldsymbol{v}-\gamma_{0} \nabla \times \nabla \times \mathbf{v} \\
+\left(\alpha_{0}+\beta_{0}+\gamma_{0}\right) \nabla(\nabla \cdot \mathbf{v})
\end{gathered}
$$

where $\mathbf{q}^{(1)}, \boldsymbol{v}$ and $p^{(1)}$ are velocity vector, microrotation vector and pressure, respectively, $\mu_{1}$ is the viscosity coefficient of the classical viscous fluid and $\kappa$ is the rotational viscosity coefficient; $\alpha_{0}, \beta_{0}$ and $\gamma_{0}$ are the gyroviscosity coefficients for the micropolar fluids.

The equations for the stress tensor $t_{i j}$ and the couple stress tensor $m_{i j}$ are

$$
\begin{gathered}
t_{i j}=-p \delta_{i j}+\mu\left(q_{i, j}+q_{j, i}\right)+\kappa\left(q_{j, i}-\varepsilon_{i j m} v_{m}\right) \\
m_{i j}=\alpha_{0} v_{m, m} \delta_{i j}+\beta_{0} v_{i, j}+\gamma_{0} v_{j, i},
\end{gathered}
$$

where the comma denotes the partial differentiation; $\delta_{i j}$ and $\varepsilon_{i j m}$ are the Kronecker delta and the alternating tensor, respectively.

For the internal region, equations of motion are

$$
\begin{gathered}
\nabla \cdot \mathbf{q}^{(2)}=0 \\
\nabla p^{(2)}+\mu_{2} \nabla \times \nabla \times \mathbf{q}^{(2)}=0
\end{gathered}
$$

where $\mathbf{q}^{(2)}$ is the velocity, $p^{(2)}$ is the pressure and $\mu_{2}$ is the coefficient of viscosity.

Let $(r, \theta, \phi)$ denote a spherical polar co-ordinate system. Since the flow of the fluid is in the meridian plane and the flow is axially symmetric, all the quantities are independent of $\phi$. Hence, we assume the velocity and microrotation vectors as

$$
\begin{gathered}
\mathbf{q}^{(i)}=q_{r}^{(i)}(r, \theta) \mathbf{e}_{r}+q_{\theta}^{(i)}(r, \theta) \mathbf{e}_{\theta}, \quad i=1,2 \\
\boldsymbol{v}=v_{\phi}(r, \theta) \mathbf{e}_{\phi}
\end{gathered}
$$

Let the surface of the spheroid be $r=a[1+f(\theta)]$. The orthogonality relations of the Gegenbauer functions $\vartheta_{m}(\zeta), \zeta=\cos \theta$ permit us, under general circumstances, to assume the expansion $f(\theta)=\sum_{m=1}^{\infty} \alpha_{m} \vartheta_{m}(\zeta)$, where the Gegenbauer function is related to the Legendre function $P_{n}(\zeta)$ by the relation

$$
\vartheta_{n}(\zeta)=\frac{P_{n-2}(\zeta)-P_{n}(\zeta)}{2 n-1}, \quad n \geq 2
$$

Therefore, the surface of the spheroid will be $r=$ $a\left[1+\alpha_{m} \vartheta_{m}(\zeta)\right]$ and assume that the coefficients $\alpha_{m}$ are sufficiently small so that their squares and higher powers may be neglected [28]. Then we have $(r / a)^{y} \approx$ $1+y \alpha_{m} \vartheta_{m}(\zeta)$ where $y$ is positive or negative.

Let $\psi^{(i)}, i=1,2$ denote the Stokes stream functions of the flow regions outside and inside the spheroid, respectively. Then the velocity components in terms of the stream function are 


$$
q_{r}^{(i)}=\frac{1}{r^{2}} \frac{\partial \psi^{(i)}}{\partial \zeta}, q_{r}^{(i)}=\frac{1}{r \sqrt{1-\zeta^{2}}} \frac{\partial \psi^{(i)}}{\partial r}, \quad i=1,2 .
$$

After elimination of the pressure $p^{(1)}$ and the microrotation vector $v_{\phi}$ from Eqs. (2)-(4) and pressure $p^{(2)}$ from (7) and (8), we obtain linear partial differential equations for the stream functions:

$$
\begin{gathered}
E^{4}\left(E^{2}-l^{2}\right) \psi^{(1)}=0 \\
E^{4} \psi^{(2)}=0
\end{gathered}
$$

with the microrotation vector given by

$$
v_{\phi}=\frac{1}{2 r \sqrt{1-\zeta^{2}}}\left(E^{2} \psi^{(1)}+\frac{2+\chi}{\chi} l^{-2} E^{4} \psi^{(1)}\right)
$$

where $E^{2}=\frac{\partial^{2}}{\partial r^{2}}+\frac{\left(1-\zeta^{2}\right)}{r^{2}} \frac{\partial^{2}}{\partial \zeta^{2}}$ is the Stokesian stream function operator, $l^{2}=\left(a^{2} \kappa(2+\chi)\right) /\left(\gamma_{0}(1+\chi)\right)$ and $\chi=\frac{\kappa}{\mu_{1}}$ is the micropolarity parameter.

\section{Boundary conditions}

To determine the flow velocity components and microrotation outside the spheroid and velocity components inside the spheroid, we assume the kinematical condition of mutual impenetrability at the surface and continuity of tangential velocity. Also, we assume as in the classical case (Happel and Brenner [28]) that the equilibrium theory of interfacial tension is applicable to our problem. This means that the presence of interfacial tension only produces a discontinuity in the normal stresses $\left(\left(\boldsymbol{n} \cdot t^{(1)}\right) \cdot \boldsymbol{n} \neq(\boldsymbol{n}\right.$. $\left.\left.t^{(2)}\right) \cdot \boldsymbol{n}\right)$ and does not in any way affect the tangential stresses. The latter is therefore continuous across the surface $\left(\left(\boldsymbol{n} \cdot t^{(1)}\right) \cdot \boldsymbol{s}=\left(\boldsymbol{n} \cdot t^{(2)}\right) \cdot \boldsymbol{s}\right)$. Hence continuity of tangential stress and non-zero spin condition for microrotation are assumed at the interface. These conditions are physically realistic and mathematically consistent (see Happel and Brenner [28], Ramkissoon [19], Ramkissoon and Majumdar [20], Niefer and Kaloni [23], Saad [24]). Therefore, the boundary conditions at the fluid surface of the spheroid are as follows:

$$
\begin{gathered}
\boldsymbol{q}^{(1)} \cdot \boldsymbol{n}=0 \\
\boldsymbol{q}^{(2)} \cdot \boldsymbol{n}=0 \\
\left(\boldsymbol{q}^{(1)}-\boldsymbol{q}^{(2)}\right) \cdot \boldsymbol{s}=0 \\
\left(\boldsymbol{n} \cdot t^{(1)}\right) \cdot \boldsymbol{s}=\left(\boldsymbol{n} \cdot t^{(2)}\right) \cdot \boldsymbol{s} \\
\boldsymbol{v}=\frac{\mathrm{s}}{2} \operatorname{curl} \boldsymbol{q}^{(2)}
\end{gathered}
$$

where $\quad \boldsymbol{n}\left(=\boldsymbol{e}_{\boldsymbol{r}}-\alpha_{m} a \nabla \vartheta_{m}(\zeta)=\boldsymbol{e}_{\boldsymbol{r}}-\alpha_{m} \sqrt{1-\zeta^{2}} P_{m-1}(\zeta)\right.$ $\left.\boldsymbol{e}_{\boldsymbol{\theta}}\right)$ is a unit normal vector, $\boldsymbol{s}$ is an arbitrary tangential vector at the spheroid surface pointing into the fluid and $s$ is a spin parameter. The value $s=0$ (no spin boundary condition) corresponds to the case where microelements close to a solid boundary are unable to rotate, whereas the value $s=1$ corresponds to the case where the microrotation is equal to the fluid vorticity at the boundary. This parameter is assumed to depend only on the nature of the fluids inside and outside the droplet.

\section{Solution of the problem}

The solution of Eq. (13) is

$$
\begin{aligned}
\psi^{(1)}= & {\left[r^{2}+b_{2} r^{-1}+d_{2} r+e_{2} \sqrt{r} K_{\frac{3}{2}}(l r)\right] \vartheta_{2}(\zeta) } \\
& +\sum_{n=3}^{\infty}\left[B_{n} r^{-n+1}+D_{n} r^{-n+3}+E_{n} \sqrt{r} K_{n-\frac{1}{2}}(l r)\right] \vartheta_{n}(\zeta) .
\end{aligned}
$$

Substituting this in Eq. (15), we get the microrotation component as

$$
\begin{aligned}
v_{\phi} & =\frac{1}{r \sqrt{1-\zeta^{2}}}\left\{\left[-d_{2} r^{-1}+l^{2} \frac{(1+\chi)}{\chi} e_{2} \sqrt{r} K_{\frac{3}{2}}(l r)\right] \vartheta_{2}(\zeta)\right. \\
& \left.+\sum_{n=3}^{\infty}\left[-(2 n-3) D_{n} r^{-n+1}+l^{2} \frac{(1+\chi)}{\chi} E_{n} \sqrt{r} K_{n-\frac{1}{2}}(l r)\right] \vartheta_{n}(\zeta)\right\}
\end{aligned}
$$

and the solution of Eq. (14) is

$$
\psi^{(2)}=\left[g_{2} r^{2}+h_{2} r^{4}\right] \vartheta_{2}(\zeta)+\sum_{n=3}^{\infty}\left[G_{n} r^{n}+H_{n} r^{n+2}\right] \vartheta_{n}(\zeta) .
$$

\section{Determination of arbitrary constants}

Substituting the expression for the unit normal and tangential vectors into Eqs. (16)-(20) gives the approximate boundary conditions (up to $\mathrm{O}\left(\alpha_{m}\right)$ ):

$$
\begin{gathered}
q_{r}^{(1)}=q_{\theta}^{(1)} \alpha_{m} \sqrt{1-\zeta^{2}} P_{m-1}(\zeta) \\
q_{r}^{(2)}=q_{\theta}^{(2)} \alpha_{m} \sqrt{1-\zeta^{2}} P_{m-1}(\zeta) \\
q_{\theta}^{(1)}=q_{\theta}^{(2)} \\
t_{r \theta}^{(1)}+\alpha_{m}\left(t_{r r}^{(1)}-t_{\theta \theta}^{(1)}\right) \sqrt{1-\zeta^{2}} P_{m-1}(\zeta) \\
=t_{r \theta}^{(2)}+\alpha_{m}\left(t_{r r}^{(2)}-t_{\theta \theta}^{(2)}\right) \sqrt{1-\zeta^{2}} P_{m-1}(\zeta)
\end{gathered}
$$




$$
v_{\phi}=\frac{s}{2} \operatorname{curl}^{(2)}
$$

The boundary conditions in terms of stream function $\psi^{(i)}, i=1,2$ on the surface of spheroid $r=1+\alpha_{m} \vartheta_{m}(\zeta)$ lead to the following relations:

$$
\begin{gathered}
\frac{\partial \psi^{(1)}}{\partial \zeta}=r \alpha_{m} \frac{\partial \psi^{(1)}}{\partial r} P_{m-1}(\zeta) \\
\frac{\partial \psi^{(2)}}{\partial \zeta}=r \alpha_{m} \frac{\partial \psi^{(2)}}{\partial r} P_{m-1}(\zeta) \\
\frac{\partial \psi^{(1)}}{\partial r}=\frac{\partial \psi^{(2)}}{\partial r} \\
2 r \frac{\partial}{\partial r}\left[\frac{1}{r}\left(\frac{\partial \psi^{(1)}}{\partial r}-\lambda \frac{\partial \psi^{(2)}}{\partial r}\right)\right]+E^{2}\left(\lambda \psi^{(2)}-\psi^{(1)}\right)-\frac{1}{l^{2}} E^{4} \psi^{(1)} \\
+2 \alpha_{m}\left[\frac{4}{r}\left(\frac{\partial^{2} \psi^{(1)}}{\partial r \partial \zeta}-\lambda \frac{\partial^{2} \psi^{(2)}}{\partial r \partial \zeta}\right)-\frac{6}{r^{2}}\left(\frac{\partial \psi^{(1)}}{\partial \zeta}-\lambda \frac{\partial \psi^{(2)}}{\partial \zeta}\right)\right. \\
\left.+\frac{P_{1}(\zeta)}{r \vartheta_{2}(\zeta)}\left(\frac{\partial \psi^{(1)}}{\partial r}-\lambda \frac{\partial \psi^{(2)}}{\partial r}\right)\right] \vartheta_{2}(\zeta) P_{m-1}(\zeta)=0 \\
v_{\phi}=\frac{s}{2 r \sqrt{1-\zeta^{2}}} E^{2} \psi^{(2)}
\end{gathered}
$$

where $\lambda=\frac{2 \sigma}{2+\chi}$ with $\sigma=\frac{\mu_{2}}{\mu_{1}}$, i.e., $\sigma$ is the classical ratio of viscosity of the internal fluid to that of the external fluid.

We first develop the solution corresponding to the boundary $r=1+\alpha_{m} \vartheta_{m}(\zeta)$. Comparison of Eqs. (21)(23) with those obtained in case of flow of an incompressible micropolar fluid past a viscous fluid sphere indicates that the terms involving $B_{n}, D_{n}, E_{n}, G_{n}$ and $H_{n}$ for $n>2$ are the extra terms here, which are not present in case of the sphere. The body that we consider is a spheroid that deviates slightly from that of a sphere and the flow generated is not expected to be very different from the one generated by flow past a fluid sphere (Niefer and Kaloni [23], Saad [24]). For the solution for the case $r=1+\sum_{m=1}^{\infty} \alpha_{m} \vartheta_{m}(\zeta)$, we employ the same technique for each $m$ and obtain the expression for stream functions in both the regions.

\section{Application to spheroid}

As a particular example of the above analysis, we now consider the particular case of flow past a prolate or oblate spheroid. The surface of the spheroid is represented in the Cartesian frame $(x, y, z)$ by the equation

$$
\frac{x^{2}+y^{2}}{c^{2}}+\frac{z^{2}}{c^{2}(1-\varepsilon)^{2}}=1
$$

where $c$ is equatorial radius and $\varepsilon$ is so small that its squares and higher powers are neglected. The polar equation of the spheroidal surface (34) is $r=1+2 \varepsilon \vartheta_{m}(\zeta)$ and $a=c(1-\varepsilon)$. For the case $0<\varepsilon \leq 1$, the spheroid is oblate, and for the case $\varepsilon<0$, it is prolate. When $\varepsilon=0$, the equation describes a sphere of radius $c$.

To apply the above results, we must take $m=2, \alpha_{m}=2 \varepsilon$. Therefore, the stream functions and the microrotation component are

$$
\begin{aligned}
\psi^{(1)}= & {\left[r^{2}+\left(b_{2}+B_{2}\right) r^{-1}+\left(d_{2}+D_{2}\right) r+\left(e_{2}+E_{2}\right) \sqrt{r} K_{\frac{3}{2}}(l r)\right] \vartheta_{2}(\zeta) } \\
& +\left[B_{4} r^{-3}+D_{4} r^{-1}+E_{4} \sqrt{r} K_{\frac{7}{2}}(l r)\right] \vartheta_{4}(\zeta)
\end{aligned}
$$

$$
\begin{aligned}
& v_{\phi}=\frac{1}{r \sqrt{1-\zeta^{2}}} \\
& \quad\left\{\left[-\left(d_{2}+D_{2}\right) r^{-1}+l^{2} \frac{(1+\chi)}{\chi}\left(e_{2}+E_{2}\right) \sqrt{r} K_{\frac{3}{2}}(l r)\right] \vartheta_{2}(\zeta)\right. \\
& \left.+\left[-5 D_{4} r^{-3}+l^{2} \frac{(1+\chi)}{\chi} E_{4} \sqrt{r} K_{\frac{7}{2}}(l r)\right] \vartheta_{4}(\zeta)\right\}
\end{aligned}
$$

$$
\begin{aligned}
\psi^{(2)}= & {\left[\left(g_{2}+G_{2}\right) r^{2}+\left(h_{2}+H_{2}\right) r^{4}\right] \vartheta_{2}(\zeta) } \\
& +\left[G_{4} r^{4}+H_{4} r^{6}\right] \vartheta_{4}(\zeta) .
\end{aligned}
$$

\section{Drag on the body}

The drag force acting on the spheroid by the external micropolar fluid can be evaluated by using the formula

$$
\mathcal{F}=\int\left(\boldsymbol{n} \cdot t^{(1)}\right) \cdot \boldsymbol{k} d S
$$

where $\mathbf{n}=\mathbf{e}_{\mathbf{r}}-\varepsilon \sin 2 \theta \mathbf{e}_{\boldsymbol{\theta}}, \quad d S=2 \pi a^{2}\left(1+2 \varepsilon \sin ^{2} \theta\right) \sin \theta \mathrm{d} \theta$, $\boldsymbol{k}$ is the unit vector in the $z$ direction and integral is taken over the surface of the body $r=1+2 \varepsilon \vartheta_{2}(\zeta)$.

$$
\begin{gathered}
\mathcal{F}=2 \pi a \mu_{1} U(2+\chi)\left(d_{2}+D_{2}\right) \\
\mathcal{F}=-\frac{12 \pi a \mu_{1} U(2+\chi)(1+\chi)}{\Delta} \\
{\left[(2+3 \sigma+\chi)(1+l)-\frac{\varepsilon}{5 \Delta}\left((1+\chi) \Delta_{1}+3 l \Delta_{2}+(2+\chi) \Delta_{3}\right)\right]}
\end{gathered}
$$

where

$$
\begin{gathered}
\Delta=6 l(1+\chi)(2+2 \sigma+\chi)+(2+\chi)(6+6 \sigma+4 \chi+5 s \chi) \\
\Delta_{1}=-6 l^{2}\left((2+\chi)^{2}-9 \sigma(2+\chi)-6 \sigma^{2}\right) \\
\Delta_{2}=6 \sigma^{2}(4+7 \chi)+\sigma(2+\chi)(36+48 \chi+5 s \chi) \\
-(2+\chi)^{2}(4+2 \chi+15 s \chi) \\
\Delta_{3}=18 \sigma^{2}-(2+\chi)(6+8 \chi+25 s \chi) \\
+\sigma(54+42 \chi+75 s \chi) .
\end{gathered}
$$




\subsection{Special cases for spheroid}

Case I When $s \rightarrow 0$, drag force acting on the fluid spheroid in the absence of spin condition is

$$
\begin{aligned}
\mathcal{F}= & -\frac{6 \pi a \mu_{1} U(2+\chi)(1+\chi)}{\Delta_{4}} \\
& {\left[(1+l)(2+3 \sigma+\chi)-\varepsilon\left(\frac{(1+\chi) \Delta_{5}+\Delta_{6}+(2+\chi) \Delta_{7}}{5 \Delta_{4}}\right)\right] }
\end{aligned}
$$

where

$$
\begin{gathered}
\Delta_{4}=3 l(1+\chi)(2+2 \sigma+\chi)+(2+\chi)(3+3 \sigma+2 \chi) \\
\Delta_{5}=-3 l^{2}\left((2+\chi)^{2}-9 \sigma(2+\chi)-6 \sigma^{2}\right) \\
\Delta_{6}=3 l\left(-(2+\chi)^{3}+6 \sigma(2+\chi)(3+4 \chi)+3 \sigma^{2}(4+7 \chi)\right) \\
\Delta_{7}=9 \sigma^{2}-(2+\chi)(3+4 \chi)+3 \sigma(9+7 \chi) .
\end{gathered}
$$

Case II When $\chi \rightarrow 0$, we get the drag force acting on the fluid spheroid when both the fluids are Newtonian:

$$
\mathcal{F}=-2 \pi a \mu_{1} U\left(\frac{2+3 \sigma}{(1+\sigma)}+\frac{\varepsilon(2-3 \sigma(3+\sigma))}{5(1+\sigma)^{2}}\right) .
$$

Case III When $\sigma \rightarrow \infty$, drag force exerted on the solid spheroid is

$$
\mathcal{F}=-6 \pi a \mu_{1} U\left(1-\frac{\varepsilon}{5}\right)
$$

\subsection{Special cases for perfect fluid sphere $(\varepsilon \rightarrow 0)$}

Case I The drag force acting on the fluid sphere in an unbounded micropolar fluid is given by

$$
\mathcal{F}=-\frac{12 \pi a \mu_{1} U(2+\chi)(1+l)(1+\chi)(2+3 \sigma+\chi)}{\Delta} .
$$

This agrees with the result of Niefer and Kaloni [23].

Case II When $s \rightarrow 0$, we get the drag force acting on the fluid sphere in the absence of spin condition on the microrotation as

$$
\mathcal{F}=-\frac{12 \pi a \mu_{1} U(2+\chi)(1+l)(1+\chi)(2+3 \sigma+\chi)}{6 l(1+\chi)(2+2 \sigma+\chi)+(2+\chi)(6+6 \sigma+4 \chi)}
$$

which agrees with the viscous fluid sphere in a micropolar fluid derived by Ramkissoon [19].

Case III If $\sigma \rightarrow \infty$, we get the drag force acting on the solid sphere as

$$
\mathcal{F}=-\frac{6 \pi a \mu_{1} U(2+\chi)(1+\chi)(1+l)}{2 l(1+\chi)+2+\chi}
$$

which agrees with the result obtained by Rao and Rao [15] and Ramkissoon and Majumdar [18].

Case IV If $\chi \rightarrow 0$, the fluid inside and outside the sphere is Newtonian. The drag force exerted on the fluid sphere by the surrounding fluid is

$$
\mathcal{F}=-\frac{2 \pi a U \mu_{1}(3 \sigma+2)}{\sigma+1}
$$

which agrees with the result obtained by Hadamard [1] and Rybczynski [2].

Equation (47) degenerates to the case of motion of a noslip solid sphere (Stokes' law) when the viscosity of the drop is infinite and to the case of motion of a perfect-slip gas bubble when the viscosity approaches zero.

\section{Results and discussion}

The variation of the drag coefficient $D_{N}\left(=\mathcal{F} /\left(6 \pi a \mu_{1} U\right)\right)$ with the classical ratio $\sigma$ of viscosity of the internal fluid (Newtonian fluid) to that of the surrounding fluid (micropolar fluid) is shown in figures 2, 3, 4, 5 for various values of the spin parameter $s$ keeping the micropolarity parameter $\chi=\frac{\kappa}{\mu_{1}}$ and the deformation parameter $\varepsilon$ as fixed and for various values of the deformation parameter $\varepsilon$ by taking the fixed values of micropolarity parameter $\chi$ and

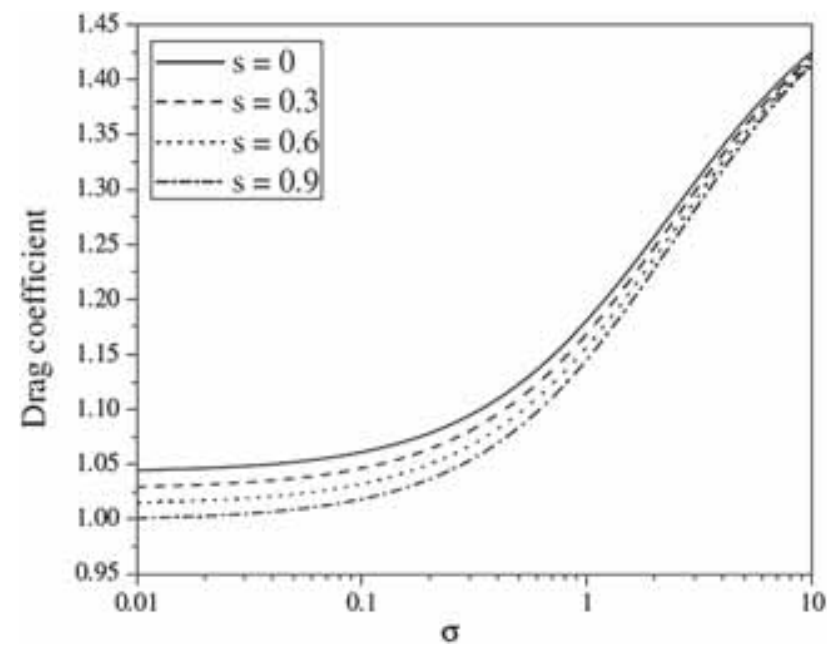

Figure 2. Variation of the drag coefficient $D_{N}$ versus viscosity ratio $\sigma$ for different values of spin parameter $s$ with the micropolarity parameter $\chi=2$ and deformation parameter $\varepsilon=0.1$. 


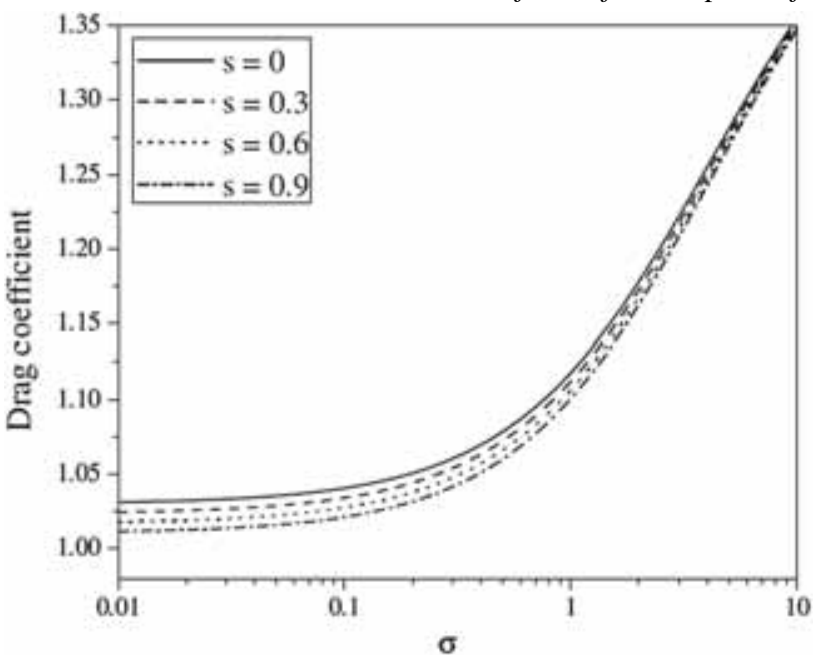

Figure 3. Variation of the drag coefficient $D_{N}$ versus viscosity ratio $\sigma$ for different values of spin parameter $s$ with the micropolarity parameter $\chi=5$ and deformation parameter $\varepsilon=0.1$.

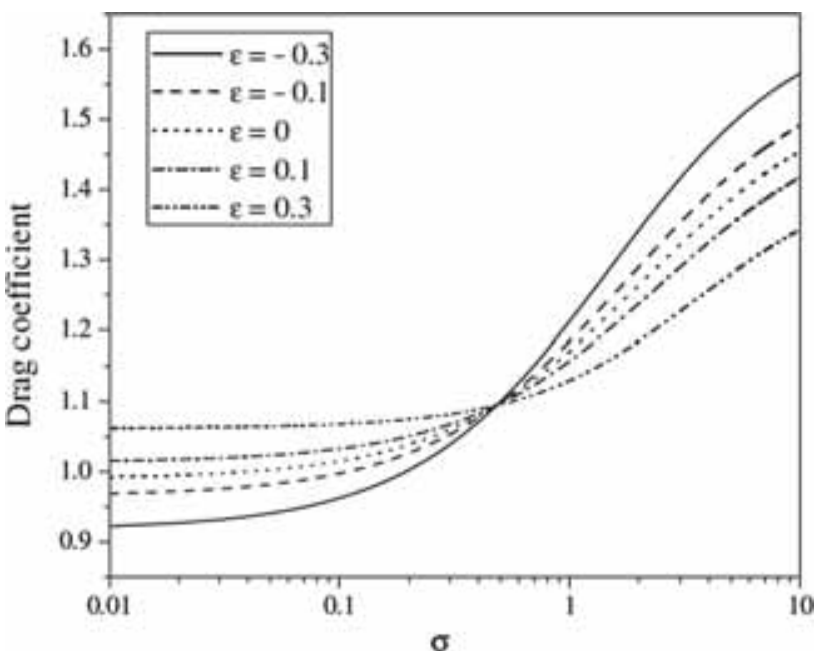

Figure 4. Variation of the drag coefficient $D_{N}$ versus viscosity ratio $\sigma$ for different values of deformity parameter $\varepsilon$ with the micropolarity parameter $\chi=2$ and spin parameter $s=0.6$.

spin parameter $s$. In numerical computation of the drag coefficient, we assumed the value $\frac{\gamma_{0}}{\mu_{1} a^{2}}=0.3$.

Figures 2 and 3 show the effect of the spin parameter $s$ on the drag coefficient $D_{N}$ with viscosity ratio $\sigma$ keeping the deformation parameter $\varepsilon(=0.1)$ as fixed and micropolarity parameter $\chi=2$ and $\chi=5$, respectively. The spin parameter ranges over the interval $0 \leq s \leq 1$. If $s=0$, there is no rotation of microelements near the boundary and if $s=1$, the microrotation is equal to the fluid vorticity at the boundary. It is observed that, in general, the drag force acting on the viscous fluid spheroid increases as viscosity ratio increases, keeping the other parameters unchanged. The drag coefficient with spin condition is less than that of no-spin condition on microrotation. As expected the drag

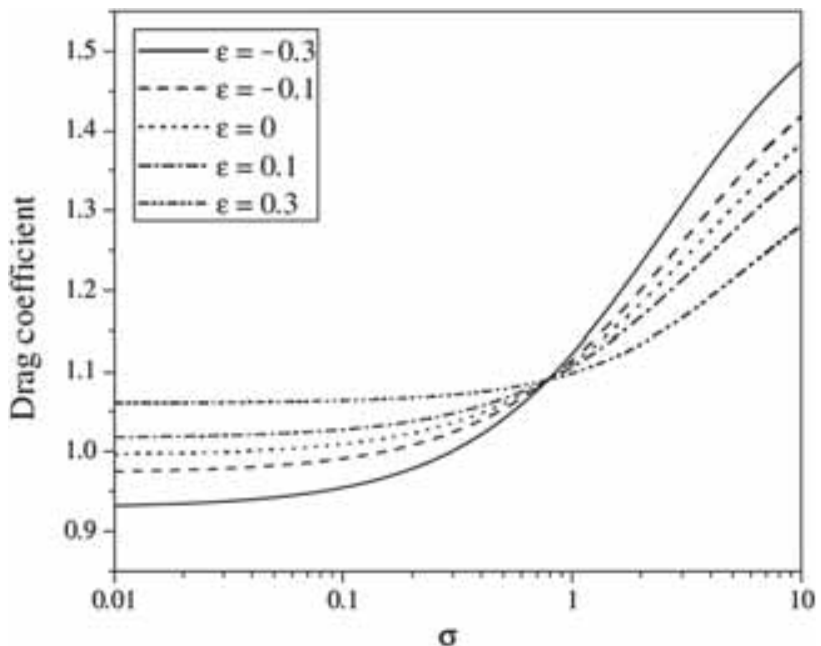

Figure 5. Variation of the drag coefficient $D_{N}$ versus viscosity ratio $\sigma$ for different values of deformity parameter $\varepsilon$ with the micropolarity parameter $\chi=5$ and spin parameter $s=0.6$.

Table 1. Drag coefficient for different values of micropolarity parameter and viscosity ratio with $\varepsilon=0.1$ and $s=0$.

\begin{tabular}{lcccc}
\hline & \multicolumn{4}{c}{ Drag coefficient } \\
\cline { 2 - 5 }$\chi$ & $\sigma=0$ & $\sigma=1$ & $\sigma=10$ & $\sigma \rightarrow \infty$ \\
\hline 0 & 0.68000 & 0.81667 & 0.94832 & 0.98000 \\
1 & 1.05122 & 1.18602 & 1.40959 & 1.47810 \\
3 & 1.72755 & 1.89912 & 2.29621 & 2.47030 \\
5 & 2.40343 & 2.59897 & 3.14808 & 3.46161 \\
7 & 3.08060 & 3.29323 & 3.97368 & 4.45270 \\
\hline
\end{tabular}

Table 2. Drag coefficient for different values of micropolarity parameter and viscosity ratio with $\varepsilon=0.1$ and $s=0.3$.

\begin{tabular}{lcccc}
\hline & \multicolumn{4}{c}{ Drag coefficient } \\
\cline { 2 - 5 }$\chi$ & $\sigma=0$ & $\sigma=1$ & $\sigma=10$ & $\sigma \rightarrow \infty$ \\
\hline 0 & 0.68000 & 0.81667 & 0.94832 & 0.98000 \\
1 & 1.03041 & 1.18410 & 1.40906 & 1.47810 \\
3 & 1.70930 & 1.89373 & 2.29421 & 2.47030 \\
5 & 2.38795 & 2.59015 & 3.14417 & 3.46161 \\
7 & 3.06701 & 3.28097 & 3.96753 & 4.45270 \\
\hline
\end{tabular}

Table 3. Drag coefficient for different values of micropolarity parameter and viscosity ratio with $\varepsilon=0.1$ and $s=1$.

\begin{tabular}{lcccc}
\hline & \multicolumn{4}{c}{ Drag coefficient } \\
\cline { 2 - 5 }$\chi$ & $\sigma=0$ & $\sigma=1$ & $\sigma=10$ & $\sigma \rightarrow \infty$ \\
\hline 0 & 0.68000 & 0.81667 & 0.94832 & 0.98000 \\
1 & 0.98485 & 1.17964 & 1.40780 & 1.47810 \\
3 & 1.66814 & 1.88127 & 2.28955 & 2.47030 \\
5 & 2.35257 & 2.56978 & 3.13507 & 3.46161 \\
7 & 3.03573 & 3.25269 & 3.95325 & 4.45270 \\
\hline
\end{tabular}


Table 4. Drag coefficient for different values of spin parameter and viscosity ratio with $\varepsilon=0.1$ and $\chi=2$.

\begin{tabular}{lcccc}
\hline & \multicolumn{4}{c}{ Drag coefficient } \\
\cline { 2 - 5 }$s$ & $\sigma=0$ & $\sigma=1$ & $\sigma=10$ & $\sigma \rightarrow \infty$ \\
\hline 0 & 1.39009 & 1.57467 & 1.90072 & 2.02281 \\
0.3 & 1.37011 & 1.55861 & 1.89537 & 2.02281 \\
0.5 & 1.35710 & 1.54808 & 1.89182 & 2.02281 \\
0.8 & 1.33803 & 1.53254 & 1.88652 & 2.02281 \\
1 & 1.32560 & 1.52236 & 1.88301 & 2.02281 \\
\hline
\end{tabular}

Table 5. Drag coefficient for different values of spin parameter and viscosity ratio with $\varepsilon=0.1$ and $\chi=5$.

\begin{tabular}{lcccc}
\hline & \multicolumn{4}{c}{ Drag coefficient } \\
\cline { 2 - 5 }$s$ & $\sigma=0$ & $\sigma=1$ & $\sigma=10$ & $\sigma \rightarrow \infty$ \\
\hline 0 & 2.40343 & 2.60858 & 3.16214 & 3.47863 \\
0.3 & 2.38795 & 2.59499 & 3.15610 & 3.47863 \\
0.5 & 2.37774 & 2.58601 & 3.15209 & 3.47863 \\
0.8 & 2.36257 & 2.57265 & 3.14608 & 3.47863 \\
1 & 2.35257 & 2.56382 & 3.14210 & 3.47863 \\
\hline
\end{tabular}

coefficient decreases with increasing spin parameter, because spin causes less motion between the fluid and the particle. As expected, the drag force exerted on the viscous fluid spheroid increases with an increase in the micropolarity parameter. Tables 1,2 and 3 show the variation of drag coefficient $D_{N}$ for deformation parameter $\varepsilon=0.1$, different values of micropolarity parameter $\chi$ and viscosity ratio for four different cases, $\sigma=0$ (fluid spheroid of vanishing viscosity), $\sigma=1$ (fluid spheroid with viscosity equal to that of the external medium), $\sigma=10$ (fluid spheroid with viscosity unequal to that of the external medium) and $\sigma \rightarrow \infty$ (rigid spheroid), for spin parameter $s=0, s=0.3$ and $s=1$, respectively. If the micropolarity parameter $\chi \rightarrow 0$, both the internal and external fluids are Newtonian and it is seen that drag coefficient increases as the viscosity ratio increases. The drag force exerted on the fluid sphere is more than that of fluid spheroid except when $\sigma=0$. Clearly, from the tables, when micropolarity parameter increases, the drag force increases as the micropolarity parameter corresponds to an extra viscous term having the asymmetric parts of the velocity gradient tensor. Tables 4 and 5 show the variation of drag coefficient $D_{N}$ for deformation parameter $\varepsilon=0.1$, different values of spin parameter $s$ and viscosity ratio for four different cases, $\sigma=0, \sigma=1, \sigma=10$ and $\sigma \rightarrow \infty$, for micropolarity parameter $\chi=2$ and $\chi=5$, respectively. It is observed that the drag coefficient decreases with increasing spin parameter.

Figures 4 and 5 depict the variation of drag coefficient $D_{N}$ with $\sigma$ for different values of deformity parameter $\varepsilon$, keeping the spin parameter $s=0.6$ as fixed and micropolarity parameter $\chi=2$ and $\chi=5$, respectively. When $\mu_{2} \ll$ $\mu_{1}$ (i.e., when $\sigma=0$ ), the problem reduces to the motion of a gas bubble rising slowly in a micropolar fluid. As $\sigma \rightarrow \infty$, the problem reduces to the motion of a solid spheroid in micropolar fluid. It is observed that the drag coefficient is an increasing or a decreasing function of deformation parameter $\varepsilon$ depending on the value of $\sigma$. For the case of gaseous bubble $\left(\mu_{2} \ll \mu_{1}\right)$, the drag coefficient is an increasing function of $\varepsilon$, whereas for the case of a solid spheroid $(\sigma \rightarrow \infty)$, it is a decreasing function of $\varepsilon$. Our numerical result for the case of solid spheroid agrees with the result previously obtained by Iyengar and Srinivasacharya [17]. Also, the drag on gaseous bubble is less than the drag on solid sphere (Happel and Brenner [28]). This is the reason why the drag is an increasing function of viscosity ratio and the behaviour of drag changes after a particular value of viscosity ratio. It is seen that the

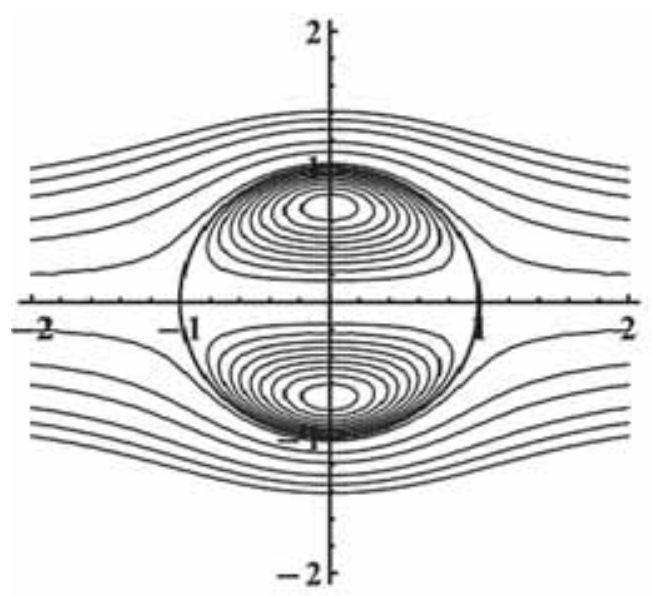

(a)

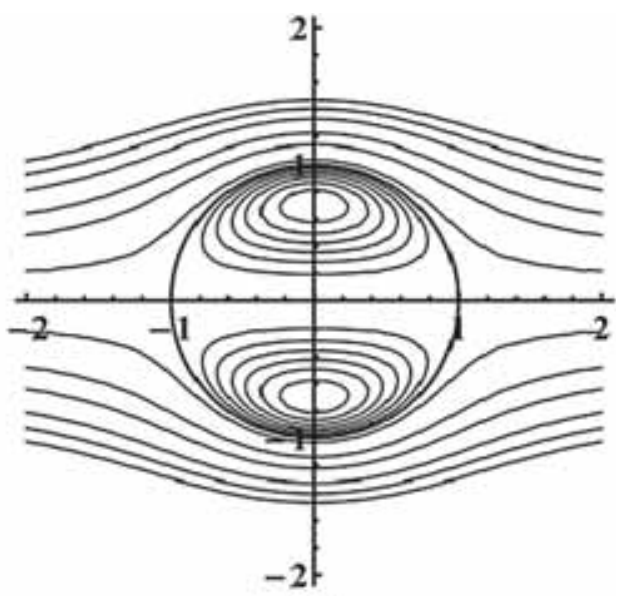

(b)

Figure 6. Streamlines for $\varepsilon=0, \chi=5$ and $s=0.6$ : (a) $\sigma=1$ and (b) $\sigma=3$. 


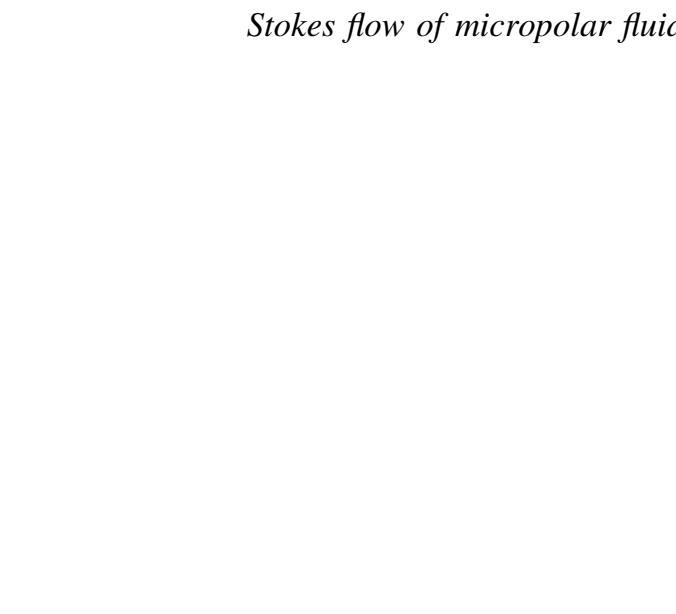

(a)

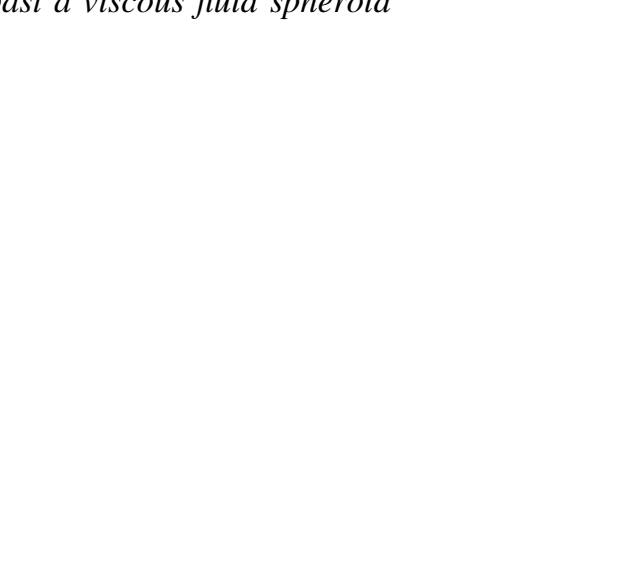

(b)

Figure 7. Streamlines for $\varepsilon=-0.03, \chi=5$ and $s=0.6$ : (a) $\sigma=1$ and (b) $\sigma=3$.

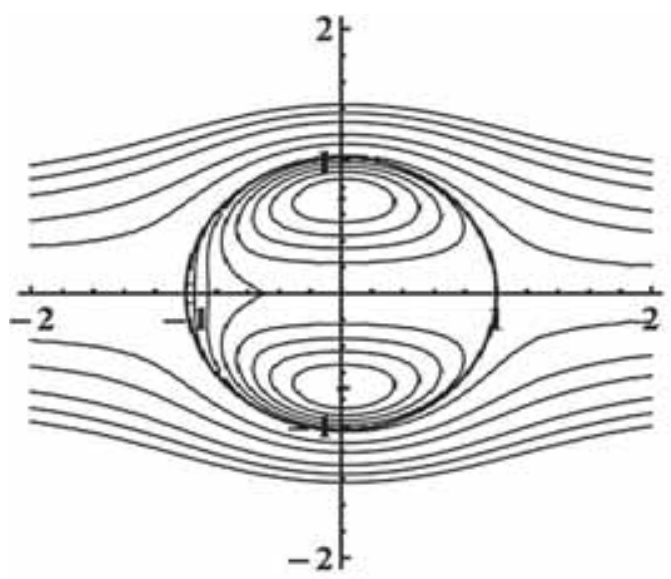

(a)

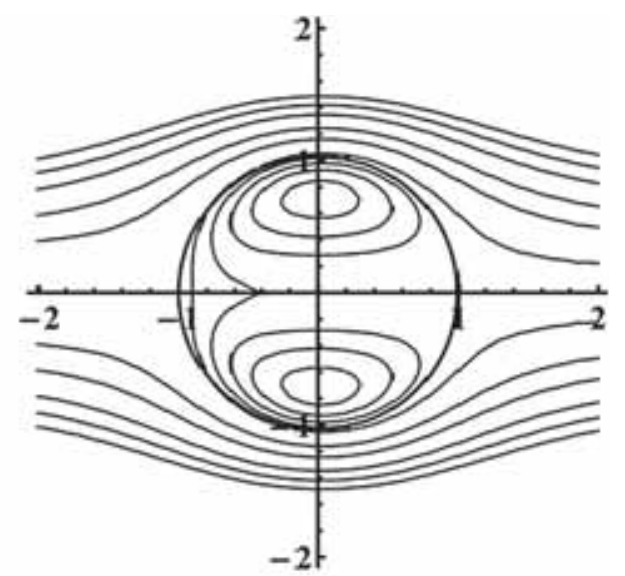

(b)

Figure 8. Streamlines for $\varepsilon=0.03, \chi=5$ and $s=0.6$ : (a) $\sigma=1$ and (b) $\sigma=3$.

behaviour of the drag coefficient changes when viscosity ratio $\sigma$ is near one. The force exerted on the fluid sphere is less than the force exerted on fluid oblate spheroid for $\sigma<1$. This is due to the fact that the external fluid is more viscous than the internal fluid.

The stream line pattern has been plotted in figures 6,7 , and 8 for different values of spin parameter $s$, micropolarity parameter $\chi$ and viscosity ratio $\sigma$ for the case of $\varepsilon=0$ (sphere), $\varepsilon=-0.03$ (prolate spheroid) and $\varepsilon=0.03$ (oblate spheroid). We observe that inside the spheroid, the Newtonian liquid executes a circulatory motion by virtue of tangential stresses that arise on the surface. It can be seen from the figures that the effect of $\chi, \sigma$ and $s$ on streamline patterns is in the circulatory motion obtained inside the spheroid.

\section{Conclusion}

An exact solution for the problem of Stokes flow of micropolar fluid past a viscous fluid spheroid is obtained by considering non-zero spin boundary condition for the microrotation vector. An expression for the drag force acting on the fluid spheroid is obtained. It is observed that the drag force is an increasing function of classical viscosity ratio of internal fluid to that of the surrounding fluid. The drag force decreases with increasing spin parameter keeping other parameters fixed. We found that the spin parameter has a significant influence on the drag. 


\section{Acknowledgements}

The authors are thankful to the reviewers for their valuable suggestions and comments.

\section{References}

[1] Hadamard J S 1911 Mouvement permanent lent d'une sphere liquide et visqueuse dans un liquide visqueux. Compt. Rend. Acad. Sci. (Paris) 152: 1735-1738

[2] Rybczynski W 1911 On the translatory motion of a fluid sphere in a viscous medium. Bull. Acad. Sci. Cracow Ser. A 40: 40-46

[3] Eringen A C 1966 Theory of micropolar fluids. J. Math. Mech. 16: 1-18

[4] Eringen A C 2001 Microcontinuum field theories II: Fluent media. New York: Springer

[5] Ariman T, Turk M A and Sylvester N D 1973 Microcontinuum fluid mechanics-A review. Int. J. Eng. Sci. 11: 905-930

[6] Ariman T, Turk M A and Sylvester N D 1974 Applications of microcontinuum fluid mechanics. Int. J. Eng. Sci. 12: 273-293

[7] Lukaszewicz G 1999 Micropolar fluids: theory and applications. Basel: Birkhäuser

[8] Hetsroni G and Haber S 1970 The flow in and around a droplet or bubble submerged in an unbounded arbitrary velocity field. Rheol. Acta 9: 488-496

[9] Bart E 1968 The slow unsteady settling of a fluid sphere toward a flat fluid interface. Chem. Eng. Sci. 23: 193-210

[10] Wacholder E and Weihs D 1972 Slow motion of a fluid sphere in the vicinity of another sphere or a plane boundary. Chem. Eng. Sci. 27: 1817-1828

[11] Lee T C and Keh H J 2012 Creeping motion of a fluid drop inside a spherical cavity. Eur. J. Mech. B: Fluids 34: 97-104

[12] Choudhuri D and Sri Padmavati B 2014 A study of an arbitrary unsteady Stokes flow in and around a liquid sphere. Appl. Math. Comp. 243: 644-656

[13] Tripathi M K, Sahu K C and Govindarajan R 2014 Why a falling drop does not in general behave like a rising bubble. Sci. Rep. 4: 4771
[14] Tripathi M K, Sahu K C and Govindarajan R 2015 Dynamics of an initially spherical bubble rising in quiescent liquid. Nat. Commun. 6: 6268

[15] Rao S K L and Rao P B 1970 The slow stationary flow of a micropolar liquid past a sphere. J. Eng. Math. 4: 209-217

[16] Rao S K L and Iyengar T K V 1981 The slow stationary flow of incompressible micropolar fluid past a spheroid. Int. J. Eng. Sci. 19: 189-220

[17] Iyengar T K V and Srinivasacharya D 1993 Stokes flow of an incompressible micropolar fluid past an approximate sphere. Int. J. Eng. Sci. 31: 115-123

[18] Ramkissoon H and Majumdar S R 1976 Drag on an axially symmetric body in the Stokes flow of micropolar fluid. Phys. Fluids 19: 16-21

[19] Ramkissoon H 1985 Flow of a micropolar fluid past a Newtonian fluid sphere. Z. Angew. Math. Mech. 65: 635-637

[20] Ramkissoon H and Majumdar S R 1988 Micropolar flow past a slightly deformed fluid sphere. Z. Angew. Math. Mech. 68: $155-160$

[21] Hayakawa H 2000 Slow viscous flows in micropolar fluids. Phys. Rev. E 61(5): 5477-5492

[22] Hoffmann K H, Marx D and Botkin N D 2007 Drag on spheres in micropolar fluids with non-zero boundary conditions for microrotations. J. Fluid Mech. 590: 319-330

[23] Niefer R and Kaloni P N 1980 On the motion of a micropolar fluid drop in a viscous fluid. J. Eng. Math. 14: 107-116

[24] Saad E I 2012 Cell models for micropolar flow past a viscous fluid sphere. Meccanica 47: 2055-2068

[25] Satya D and Pankaj S 2012 Creeping flow of micropolar fluid past a fluid sphere with non-zero spin boundary condition. Int. J. Eng. Technol. 1(2): 67-76

[26] Jaiswal B R and Gupta B R 2014 Drag on Reiner-Rivlin liquid sphere placed in a micropolar fluid with non-zero boundary condition for microrotations. Int. J. Appl. Math. Mech. 10(7): 90-103

[27] Faltas M S and Saad E I 2014 Slow motion of spherical droplet in a micropolar fluid flow perpendicular to a planar solid surface. Eur. J. Mech. B: Fluids 48: 266-276

[28] Happel J and Brenner H 1965 Low Reynolds number hydrodynamics. Englewood Cliffs, NJ: Prentice-Hall 